Supporting Information

\title{
The Construction of Hydrangea-like Vanadium Doped Iron Nickel Phosphide as an Enhanced Bifunctional Electrocatalyst for Overall Water Splitting
}

Na Suo ${ }^{a}$, Cheng Chen ${ }^{a}$, Xinqi Han ${ }^{a}$, Xingquan He ${ }^{a}$, Zhiyu Dou ${ }^{a}$, Zihan Lin $^{a}$, Lili Cui* ${ }^{a}$, Jinbao Xiang*b

a. Key Laboratory of Applied Chemistry and Nanotechnology at Universities of Jilin Province, School of Chemistry and Environmental Engineering, Changchun University of Science and Technology, Changchun 130022, P.R. China

b. The Center for Combinatorial Chemistry and Drug Discovery of Jilin University, The School of Pharmaceutical Sciences, Jilin University, 1266 Fujin Road, Changchun, Jilin 130021, P.R. China

*E-mail: cuilili@cust.edu.cn; jbxiang@jlu.edu.cn (Jinbao Xiang) 


\section{Structure and Surface Characterization}

A JEOL JSM-6701F scanning electron microscopy (SEM) at $10 \mathrm{kV}$ and a JEOL-2010 transmission electron microscopy (TEM) at $200 \mathrm{kV}$ were used to investigate the morphologies. $\mathrm{X}$-ray diffraction $(\mathrm{XRD})$ patterns were recorded on a powder X-ray diffractometer under $40 \mathrm{kV}$ and $15 \mathrm{~mA}$ using $\mathrm{Cu}-\mathrm{Ka}$ radiation (RIGAKU, D/MAX2550VB/PC). An ESCLAB 250 spectrometer with a monochromatized Al Ka X-ray source was carried out to measure the X-ray photoelectron spectroscopy (XPS).

\section{Electrochemical Measurements}

All the HER and OER tests were carried on a CHI 760E electrochemical workstation with a three-electrode electrochemical cell using graphite rod as the counter electrode (CE), saturated calomel electrode (SCE) as the reference electrode (RE) and the electrocatalyst supported on the $\mathrm{NF}$ as the working electrode (WE). Water splitting tests were carried on a $\mathrm{CHI} 760 \mathrm{E}$ electrochemical workstation and DC power with a two-electrode electrochemical system. The freshly prepared samples were used as working electrodes.

\section{Calculation of sample load}

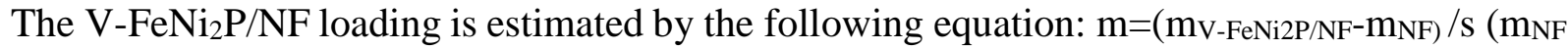
present the mass of NF. $\mathrm{m}_{\mathrm{V}-\mathrm{FeNi} 2 \mathrm{P} / \mathrm{NF}}$ is the mass of $\mathrm{FeNi}_{2} \mathrm{P}$ and NF. $\mathrm{m}$ represents mass per unit area, $\mathrm{s}$ represents catalyst area.) In our case, the load calculation of the $\mathrm{V}-\mathrm{FeNi}_{2} \mathrm{P} / \mathrm{NF}$ sample is about $0.4 \mathrm{mg} \mathrm{cm}^{-2}$.

Preparation of contrast samples $\mathbf{P t} / \mathbf{C} / \mathbf{N F}$ and $\mathrm{RuO}_{2} / \mathrm{NF}$ : Typically, the $\mathrm{Pt} / \mathrm{C}$ and $\mathrm{RuO}_{2}$ powder $(1 \mathrm{mg})$ was dispersed in $1 \mathrm{~mL}$ ethanol, respectively. The mixture was then ultrasonicated 
for $30 \mathrm{~min}$ to generate a homogeneous ink. Next, the ink $(0.1 \mathrm{~mL})$ was coating on $\mathrm{Ni}$ foam $(0.25$ $\left.\mathrm{cm}^{2}\right)$ by microsyringe and glued by $0.01 \mathrm{~mL}$ nafion $(0.5 \mathrm{wt} \%)$, then the as-prepared film was dried at room temperature. The loading of $\mathrm{Pt} / \mathrm{C}$ and $\mathrm{RuO}_{2}$ on $\mathrm{NF}$ is $0.4 \mathrm{mg} \mathrm{cm}{ }^{-2}$.

The ECSA of all samples were evaluated by the electrochemical double-layer capacitance derived from $\mathrm{CV}$ test, the $\mathrm{CV}$ test were conducted at different scan rate $\left(0-300 \mathrm{mV} \mathrm{s} \mathrm{s}^{-1}\right)$ in a potential of 0.2 to $0.3 \mathrm{~V}$ vs. RHE. The current density variation at $0.25 \mathrm{vs}$. RHE was drew against the scan rate. Electrochemical impedance spectroscopy (EIS) was measured at the open-circuit voltage from $100 \mathrm{kHz}$ to $0.1 \mathrm{~Hz}$ with a $5 \mathrm{mV} \mathrm{AC}$ dither in $1.0 \mathrm{M} \mathrm{KOH}$. Linear sweep voltammetry (LSV) measurements were performed for HER at the scan rate of $1 \mathrm{mV} \mathrm{s}^{-1}$ and OER at the scan rate of $0.1 \mathrm{mV} \mathrm{s}^{-1}$, all $\mathrm{LSV}$ tests without any iR compensation in $1.0 \mathrm{M} \mathrm{KOH}$. Cyclic voltammograms $(\mathrm{CV})$ measurements were conducted at a scan rate of $100 \mathrm{mV} \mathrm{s}^{-1}$. All the polarization curves were obtained after a stable multi-circle CV test, the potentials were showed versus reversible hydrogen electrode (RHE) by equation of $E_{v s . R H E}=E_{v s . S C E}+E_{S C E}^{\theta}+0.059 p H$. 


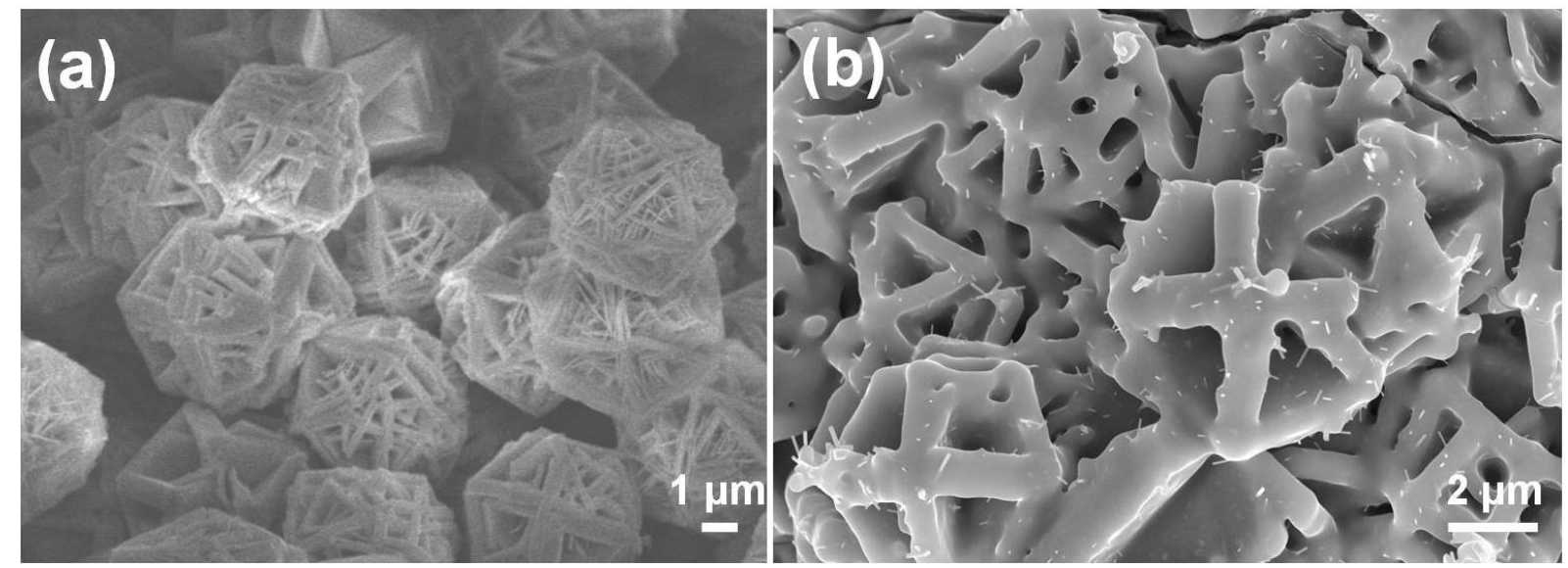

Figure S1. SEM image of (a) FeNi-LDH/NF and (b) FeNi $2 \mathrm{P} / \mathrm{NF}$ images.
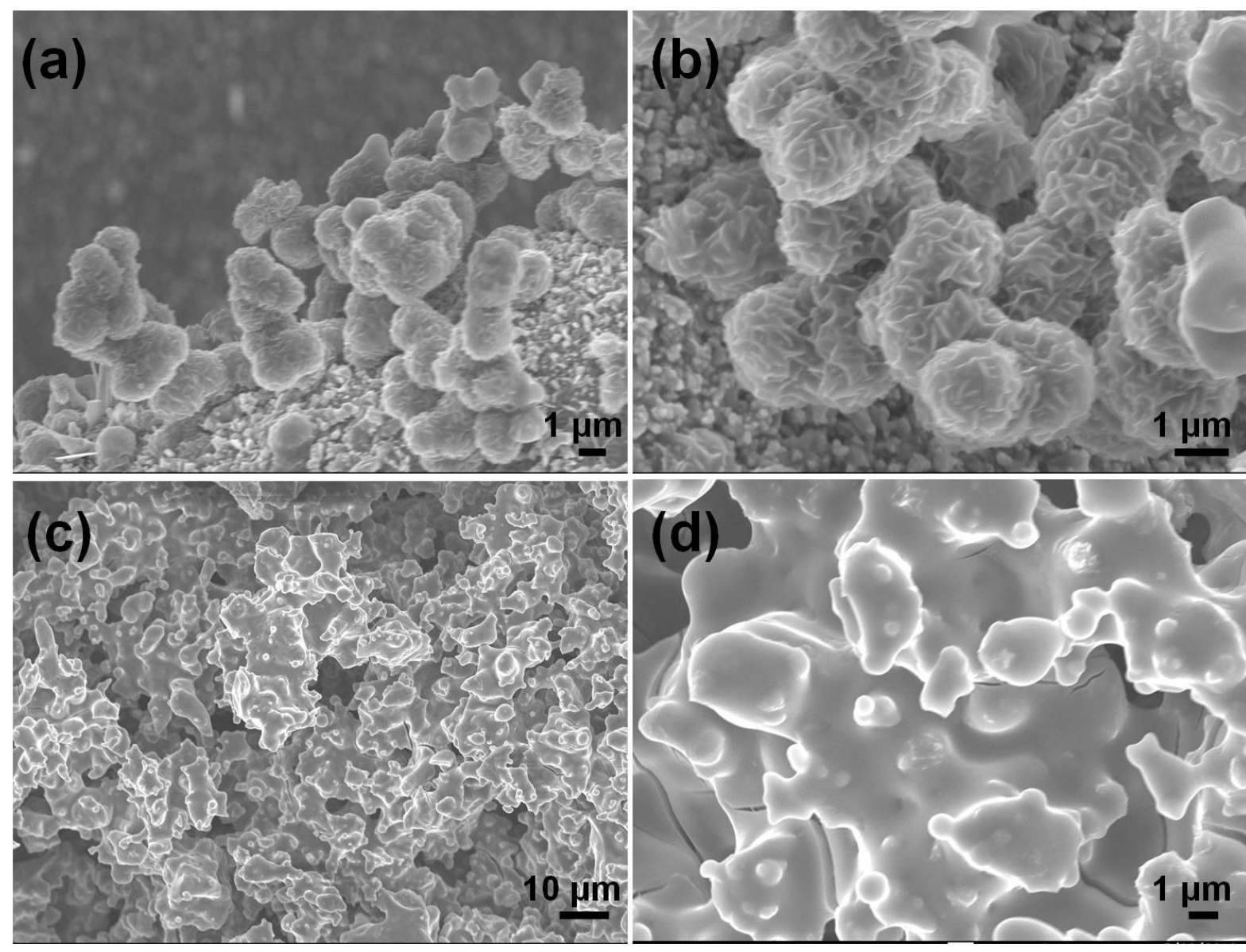

Figure S2. SEM images of $(\mathrm{a}, \mathrm{b}) \mathrm{V}-\mathrm{FeNi}{ }_{2} \mathrm{P} / \mathrm{NF}\left(\mathrm{NaH}_{2} \mathrm{PO}_{2}, 150 \mathrm{mg}\right)$ and (c, d) $\mathrm{V}-\mathrm{FeNi} \mathrm{P}_{2} \mathrm{P} / \mathrm{NF}$ $\left(\mathrm{NaH}_{2} \mathrm{PO}_{2}, 350 \mathrm{mg}\right)$ 

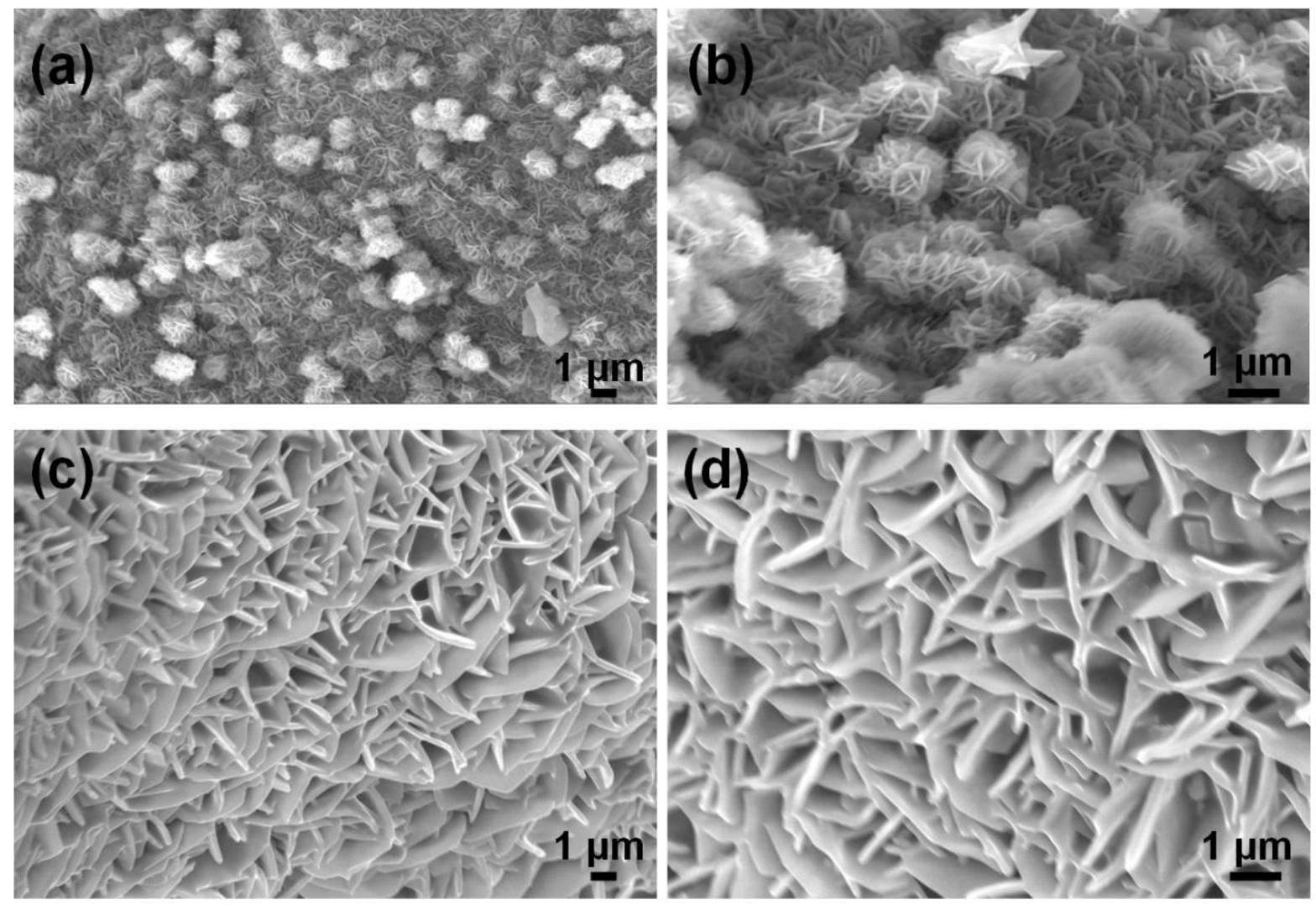

Figure S3. SEM image of (a, b) $\mathrm{V}-\mathrm{FeNi}_{2} \mathrm{P} / \mathrm{NF}-0$ and $(\mathrm{c}, \mathrm{d}) \mathrm{V}-\mathrm{FeNi}_{2} \mathrm{P} / \mathrm{NF}-4$.
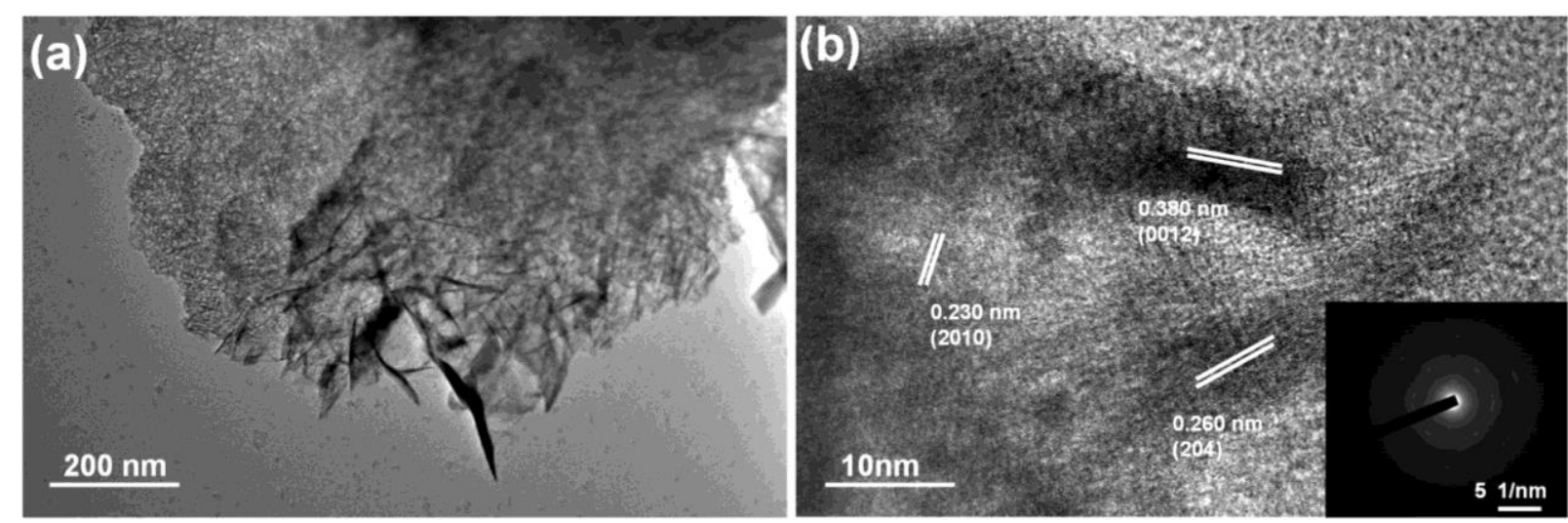

Figure S4. (a) TEM images and (b) HR-TEM images of V-FeNi-LDH/NF. Inset: SAED image of V-FeNi-LDH/NF. 

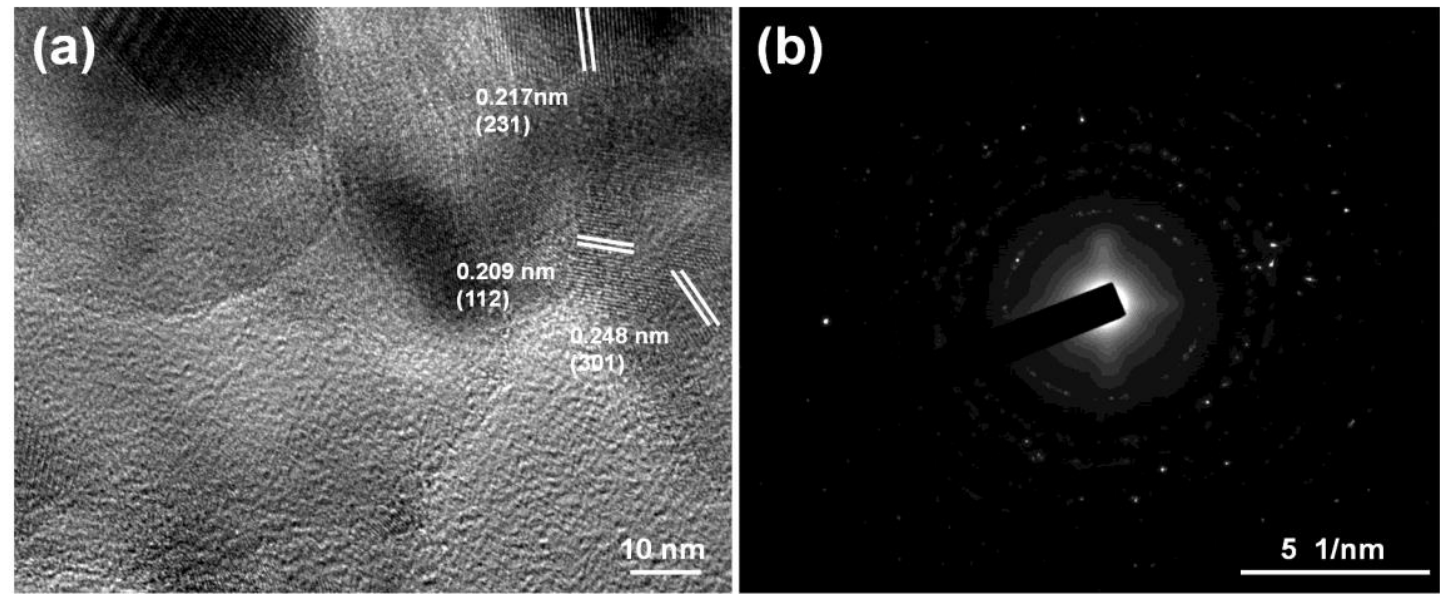

Figure S5. (a) HR-TEM images of $\mathrm{FeNi}_{2} \mathrm{P} / \mathrm{NF}$. (b)The SAED images of $\mathrm{FeNi}_{2} \mathrm{P} / \mathrm{NF}$.
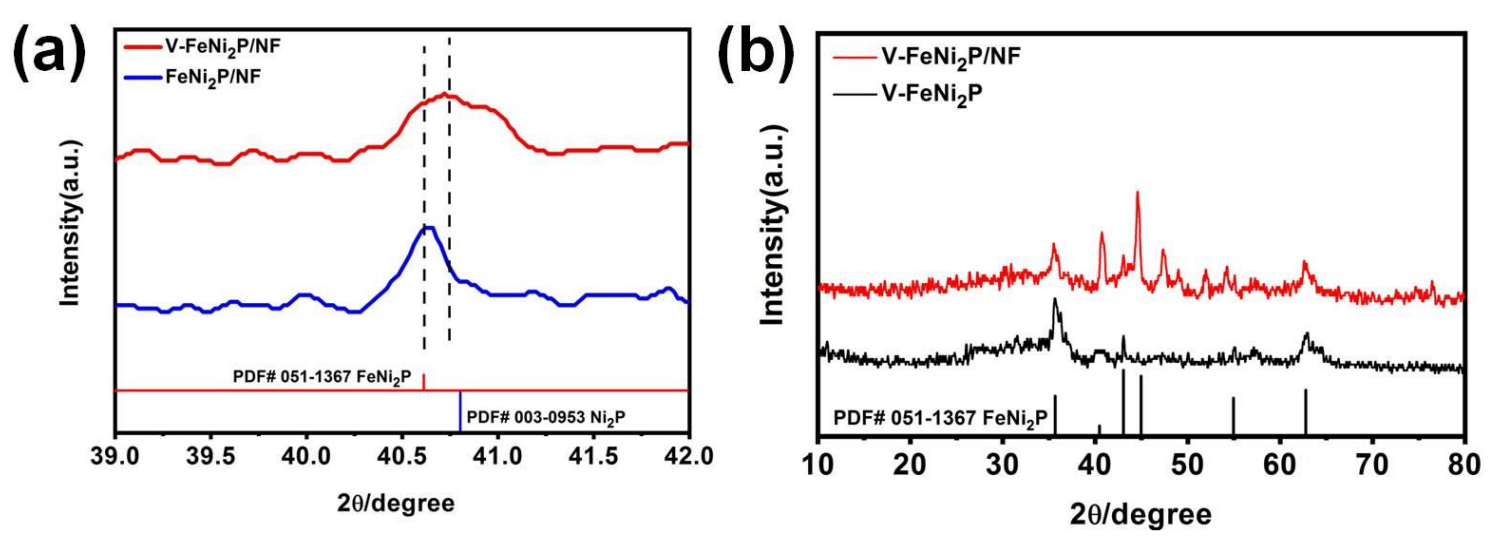

Figure S6. (a) XRD spectra for $\mathrm{V}-\mathrm{FeNi}_{2} \mathrm{P} / \mathrm{NF}$ and $\mathrm{FeNi}_{2} \mathrm{P} / \mathrm{NF}$ at $39^{\circ}$ to $42^{\circ}$. (b) XRD spectra for $\mathrm{V}-\mathrm{FeNi}_{2} \mathrm{P}$ and $\mathrm{V}-\mathrm{FeNi}_{2} \mathrm{P} / \mathrm{NF}$. 
Table S1. Elemental content of samples measured by XPS.

\begin{tabular}{lcccccccc}
\hline Sample/Element & $\mathrm{Ni}$ & $\mathrm{Fe}$ & $\mathrm{V}$ & $\mathrm{P}$ & $\mathrm{O}$ & $\mathrm{C}$ & $\mathrm{N}$ & $\mathrm{F}$ \\
\hline V-FeNi-LDH/NF & 8.86 & 3.75 & 2.67 & $/$ & 35.53 & 41.62 & 2.86 & 4.71 \\
V-FeNi 2 P/NF & 6.14 & 3.19 & 0.61 & 16.17 & 42.02 & 28.14 & 2.92 & 0.8 \\
After 5000 CV HER & 6.29 & 3.16 & 0.52 & 3.74 & 40.7 & 42.22 & 2.09 & 1.27 \\
After 5000 CV OER & 6.09 & 3.3 & 0.45 & 1.95 & 42.8 & 41.56 & 3.0 & 0.84 \\
After 10000 CV OER & 6.08 & 3.77 & 0.21 & 0.85 & 45.55 & 41.07 & 1.52 & 0.95 \\
\hline
\end{tabular}
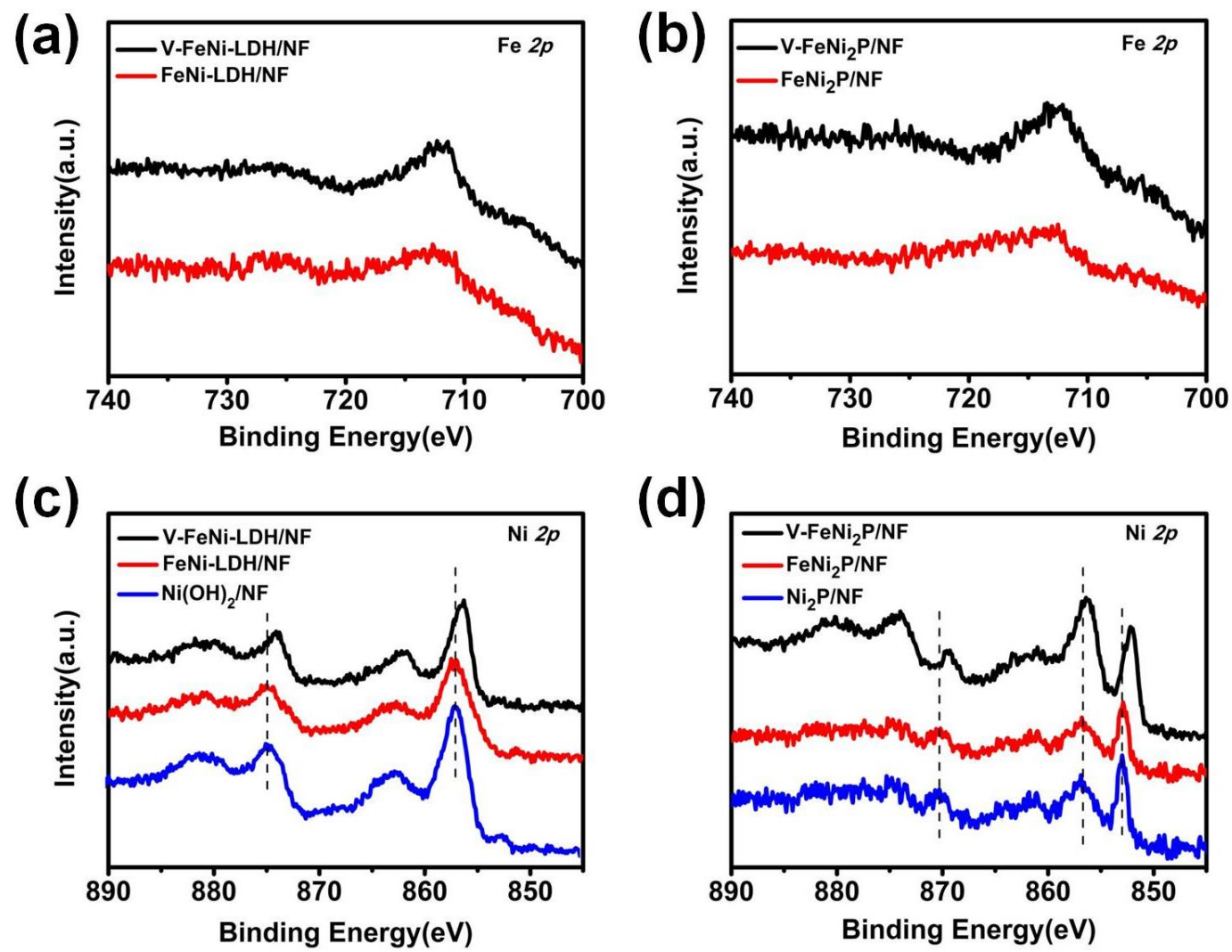

Figure S7. (a) XPS spectra of Fe for FeNi-LDH/NF and V-FeNi-LDH/NF. (b) XPS spectra of Fe for $\mathrm{FeNi}_{2} \mathrm{P} / \mathrm{NF}$ and $\mathrm{V}-\mathrm{FeNi}_{2} \mathrm{P} / \mathrm{NF}$. (c) $\mathrm{XPS}$ spectra of $\mathrm{Ni}$ for $\mathrm{Ni}(\mathrm{OH})_{2} / \mathrm{NF}, \mathrm{FeNi}-\mathrm{LDH} / \mathrm{NF}$ and V-FeNi-LDH/NF. (d) XPS spectra of Ni for $\mathrm{Ni}_{2} \mathrm{P} / \mathrm{NF}, \mathrm{FeNi}_{2} \mathrm{P} / \mathrm{NF}$ and $\mathrm{V}-\mathrm{FeNi}_{2} \mathrm{P} / \mathrm{NF}$. 
(a)

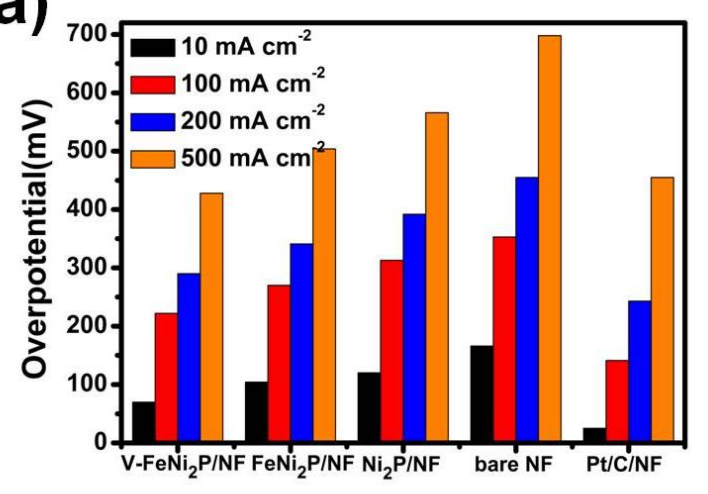

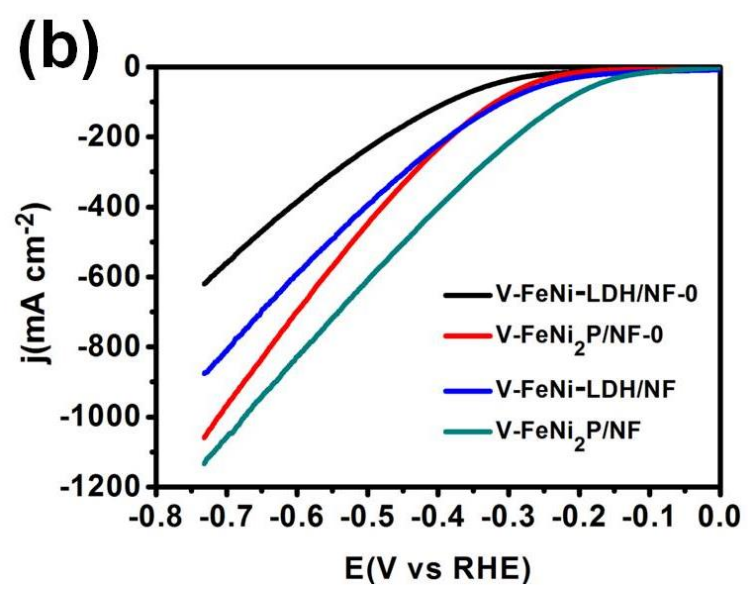

Figure S8. (a) Overpotential required for $\mathrm{j}=10,100,200$ and $500 \mathrm{~mA} \mathrm{~cm}^{-2}$ of $\mathrm{V}-\mathrm{FeNi}{ }_{2} \mathrm{P} / \mathrm{NF}$, $\mathrm{FeNi}_{2} \mathrm{P} / \mathrm{NF}, \mathrm{Ni}_{2} \mathrm{P} / \mathrm{NF}$, bare $\mathrm{NF}$ and $\mathrm{Pt} / \mathrm{C} / \mathrm{NF}$ in $1.0 \mathrm{M} \mathrm{KOH}$ with a scan rate of $1 \mathrm{mV} \mathrm{s}^{-1}$ at room temperature. (b) LSV curves of V-FeNi-LDH/NF-0, V-FeNi 2 P/NF-0, V-FeNi-LDH/NF and $\mathrm{V}-\mathrm{FeNi}_{2} \mathrm{P} / \mathrm{NF}$ for HER.

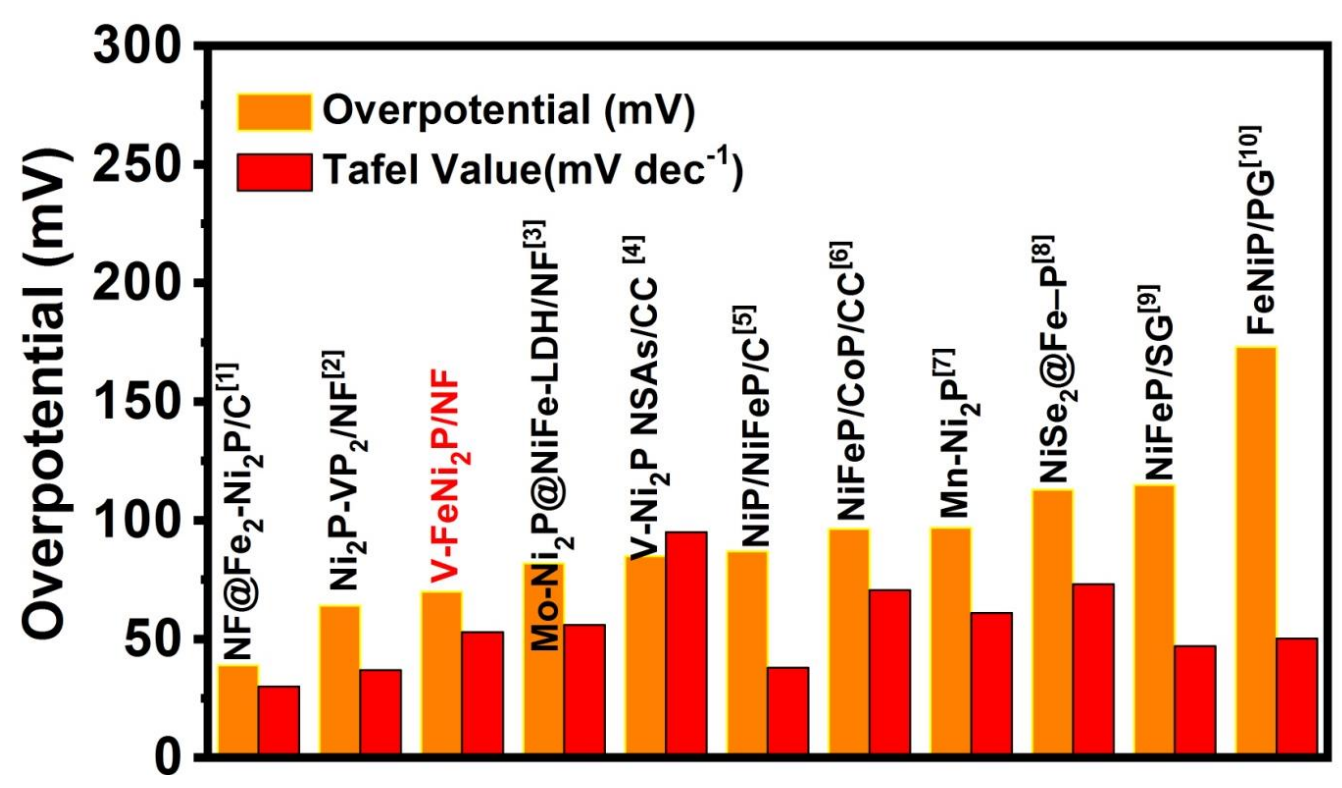

Figure S9. Comparison of the LSV and Tafel value of the V-FeNi $2 \mathrm{P} / \mathrm{NF}$ to other reported TMPs high performance for HER in $1 \mathrm{M} \mathrm{KOH}$. 
Table S2. HER reversibility data based on $\mathrm{V}-\mathrm{FeNi}_{2} \mathrm{P} / \mathrm{NF}$ with current densities from 10 to $500 \mathrm{~mA}$ $\mathrm{cm}^{-2}$ in $1 \mathrm{M} \mathrm{KOH}$.

\begin{tabular}{cccc}
\hline$j\left(\mathrm{~mA} \mathrm{~cm}^{-2}\right)$ & $\eta(\mathrm{mV})$-start & $\eta(\mathrm{mV})$-end & $|\triangle \mathrm{E}|(\mathrm{mV})$ \\
\hline 10 & -0.084 & -0.087 & 0.003 \\
20 & -0.109 & -0.111 & 0.002 \\
50 & -0.160 & -0.158 & 0.002 \\
100 & -0.201 & -0.204 & 0.003 \\
200 & -0.249 & -0.251 & 0.002 \\
500 & & -0.341 &
\end{tabular}



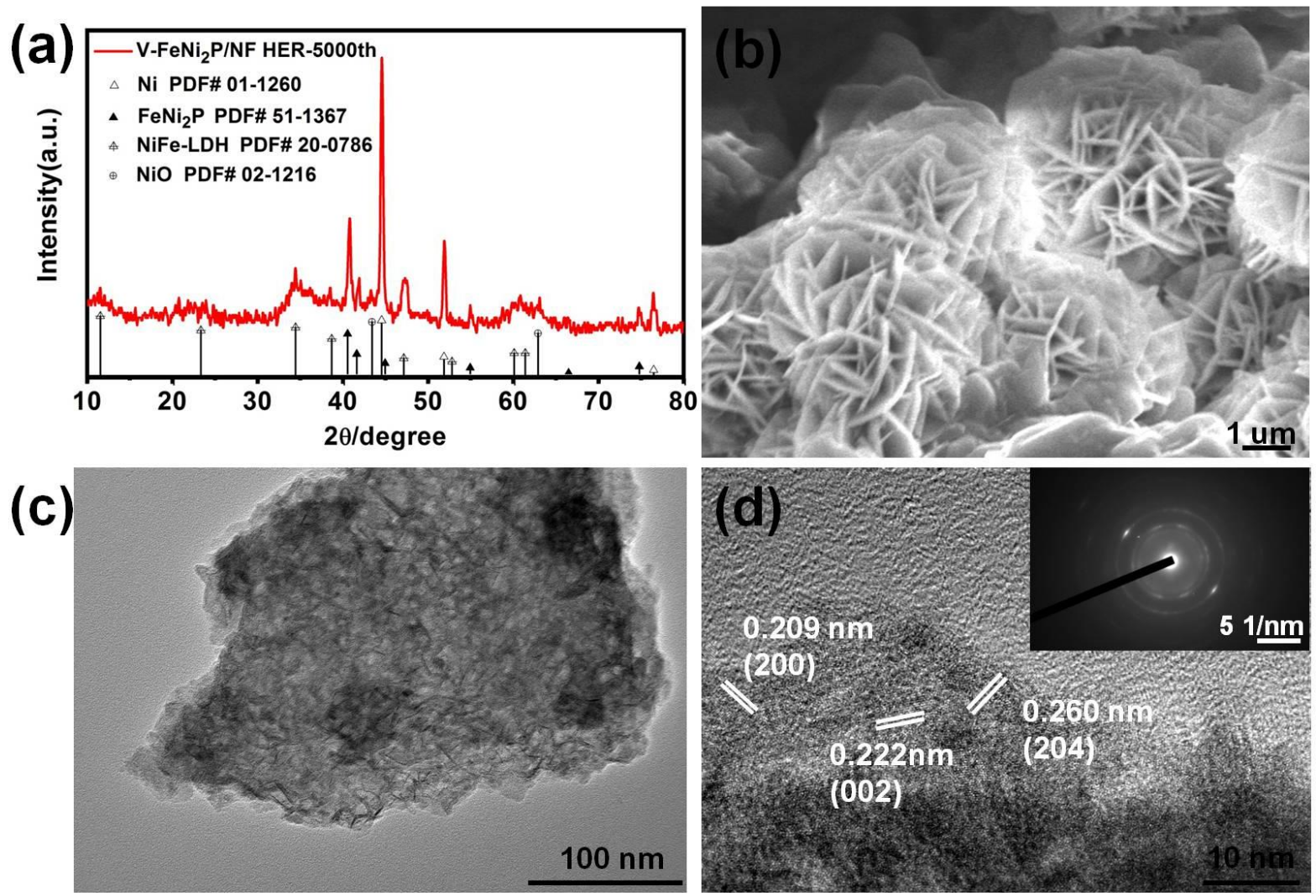

Figure S10. (a) XRD Pattern, (b) SEM images, (c) TEM images and (d) HR-TEM images of

$\mathrm{V}-\mathrm{FeNi}_{2} \mathrm{P} / \mathrm{NF}$ after the HER test. Inset: SAED images of $\mathrm{V}-\mathrm{FeNi}{ }_{2} \mathrm{P} / \mathrm{NF}$ after the HER test. 
(a)

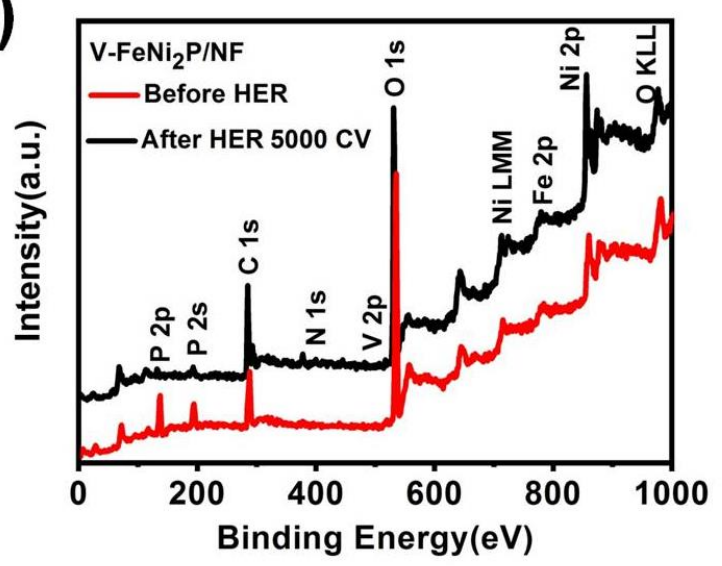

(b)

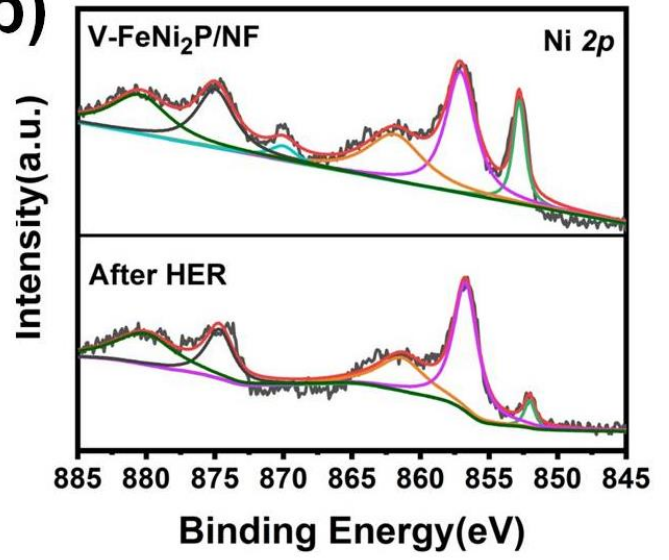

Figure S11. (a) XPS spectra and (b) high resolution XPS spectra of Ni for $\mathrm{V}-\mathrm{FeNi}_{2} \mathrm{P} / \mathrm{NF}$ after the HER test.

(a)

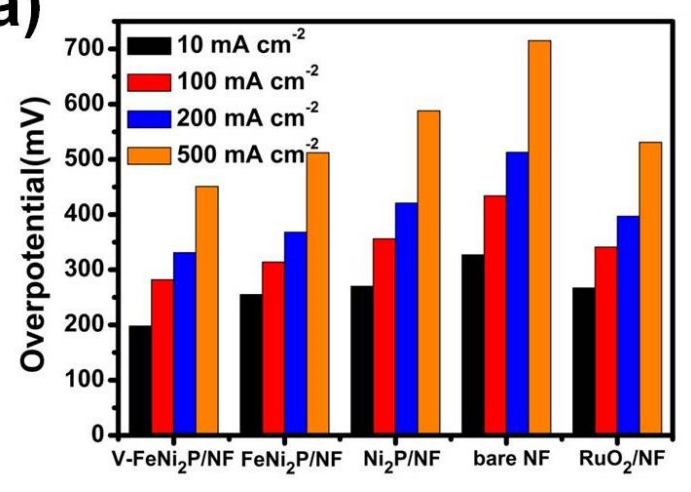

(b)

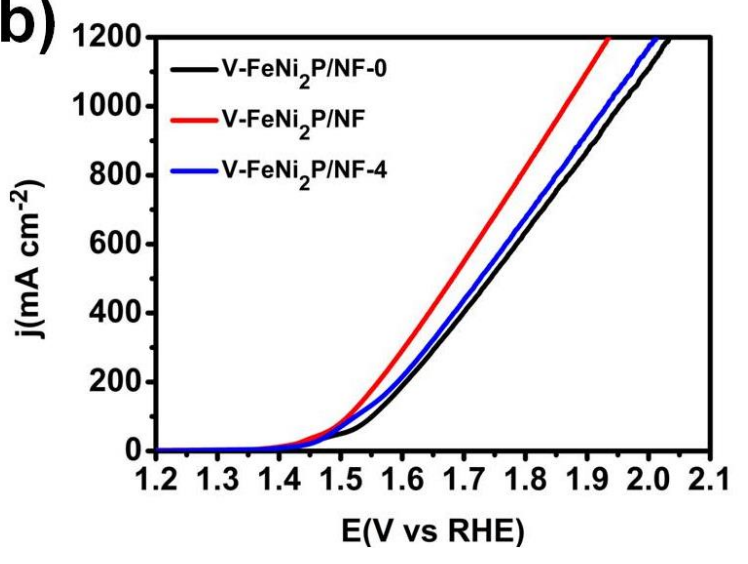

Figure S12. (a) Overpotential required for $\mathrm{j}=10,100,200$ and $500 \mathrm{~mA} \mathrm{~cm}{ }^{-2}$ of $\mathrm{V}-\mathrm{FeNi} 2 \mathrm{P} / \mathrm{NF}$,

$\mathrm{FeNi}_{2} \mathrm{P} / \mathrm{NF}, \mathrm{Ni}_{2} \mathrm{P} / \mathrm{NF}$, bare $\mathrm{NF}$ and $\mathrm{RuO}_{2} / \mathrm{NF}$ in $1.0 \mathrm{M} \mathrm{KOH}$ with a scan rate of $0.1 \mathrm{mV} \mathrm{s}^{-1}$ at room temperature. (b) LSV curves of $\mathrm{V}-\mathrm{FeNi}_{2} \mathrm{P} / \mathrm{NF}-0, \mathrm{~V}-\mathrm{FeNi} \mathrm{i}_{2} \mathrm{P} / \mathrm{NF}$ and $\mathrm{V}-\mathrm{FeNi}_{2} \mathrm{P} / \mathrm{NF}-4$ for OER. 


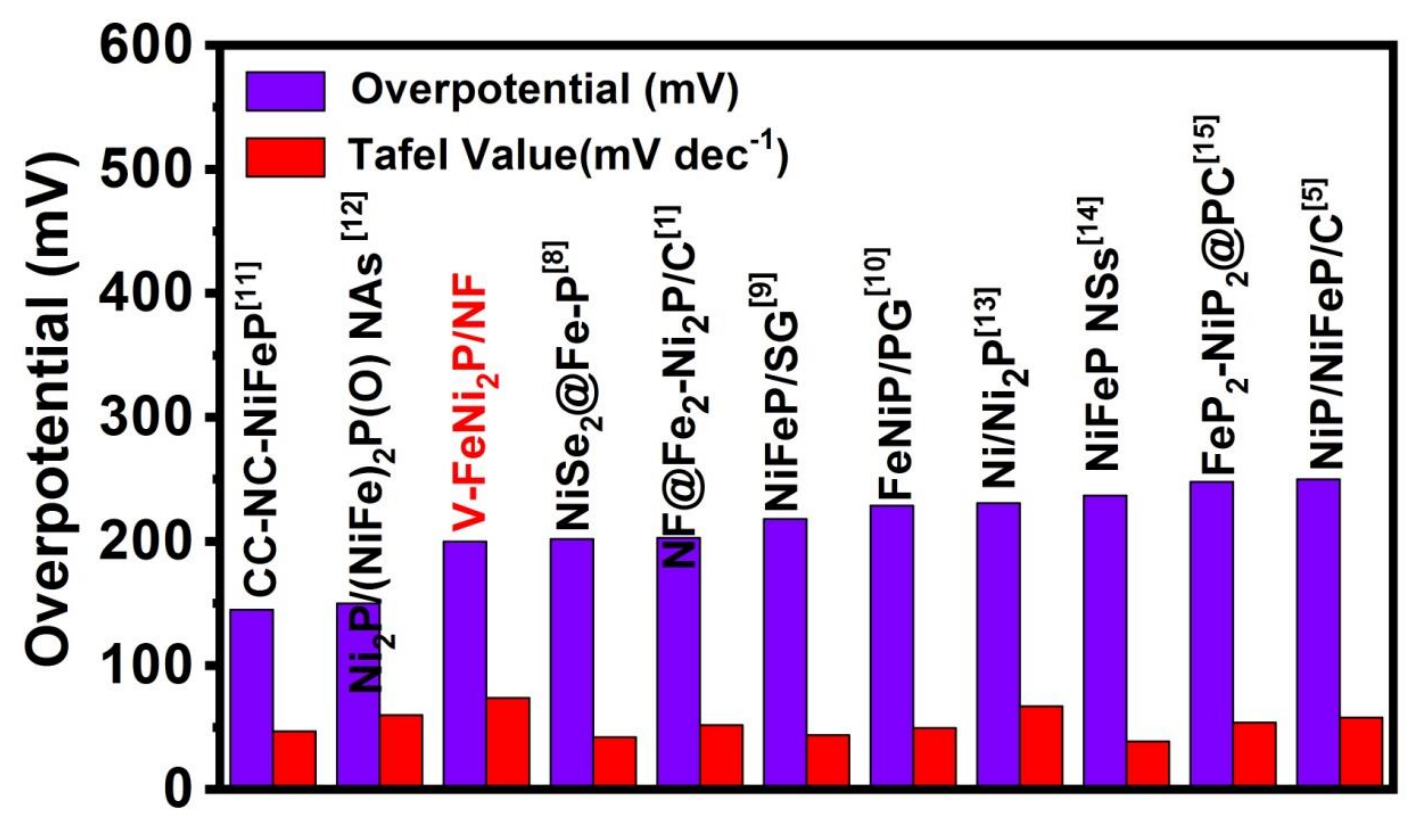

Figure S13. Comparison of the LSV and Tafel value of the $\mathrm{V}-\mathrm{FeNi}_{2} \mathrm{P} / \mathrm{NF}$ to other reported TMPs high performance for OER in $1 \mathrm{M} \mathrm{KOH}$.

Table S3. OER reversibility data based on $\mathrm{V}-\mathrm{FeNi}_{2} \mathrm{P} / \mathrm{NF}$ with current densities from 10 to $500 \mathrm{~mA}$ $\mathrm{cm}^{-2}$ in $1 \mathrm{M} \mathrm{KOH}$.

\begin{tabular}{cccc}
\hline $\mathrm{j}\left(\mathrm{mA} \mathrm{cm}^{-2}\right)$ & $\eta(\mathrm{mV})$-start & $\eta(\mathrm{mV})$-end & $|\triangle \mathrm{E}|(\mathrm{mV})$ \\
\hline 10 & 1.4373 & 1.4392 & 0.0019 \\
20 & 1.4556 & 1.4551 & 0.0005 \\
50 & 1.4812 & 1.4811 & 0.0001 \\
100 & 1.5121 & 1.5144 & 0.0023 \\
200 & 1.5674 & & 1.5676 \\
500 & & 1.6882 & \\
\hline
\end{tabular}


(a)

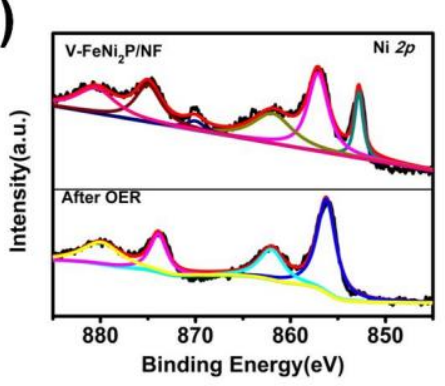

(b)

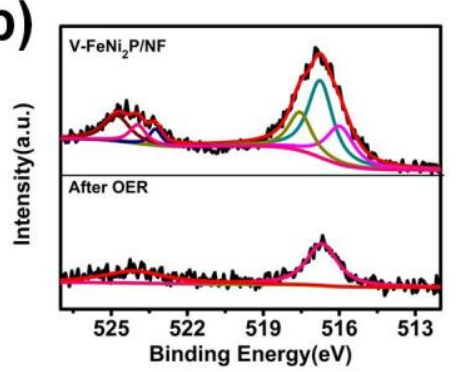

(c)

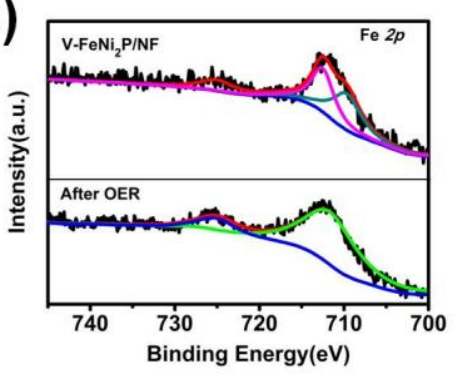

(d)

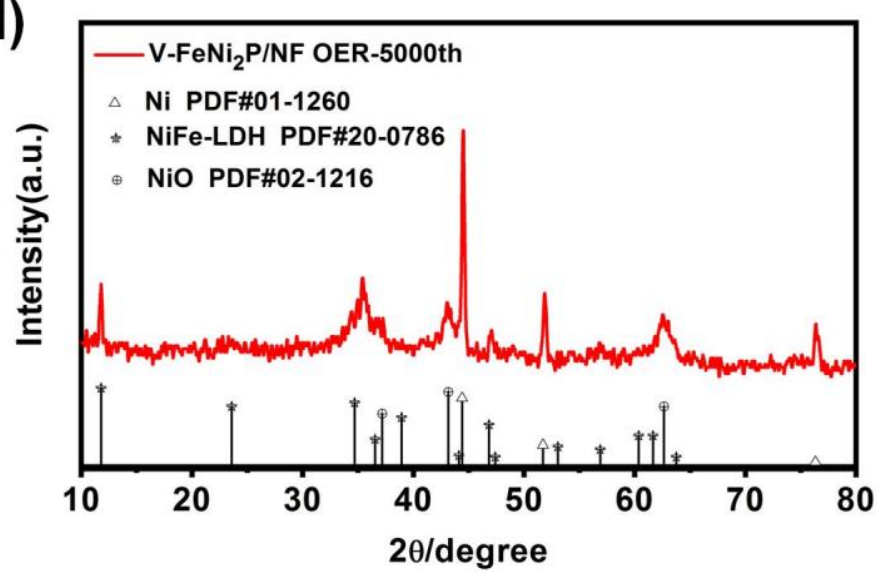

Figure S14. (a-c) High resolution XPS spectra of Ni, V, Fe for V-FeNi $2 \mathrm{P} / \mathrm{NF}$ after the OER test.

(d) XRD Pattern of $\mathrm{V}-\mathrm{FeNi}_{2} \mathrm{P} / \mathrm{NF}$ after the OER test.
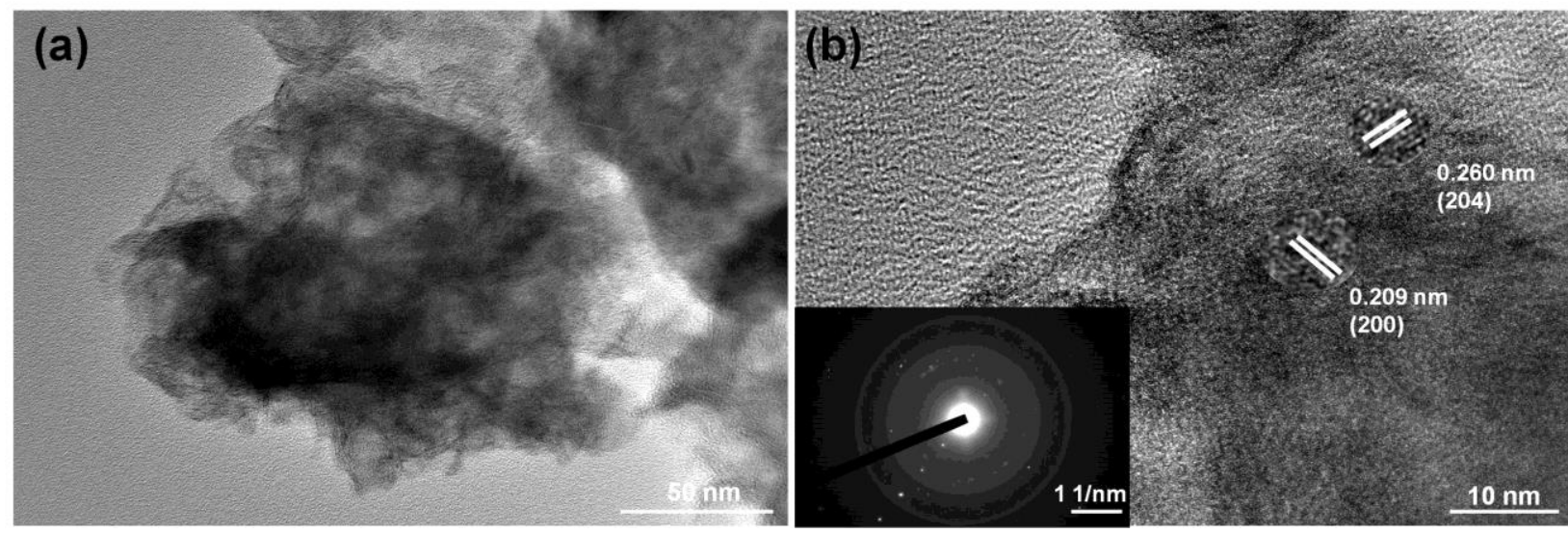

Figure S15. (a) TEM images and (b) HR-TEM images of $\mathrm{V}-\mathrm{FeNi}_{2} \mathrm{P} / \mathrm{NF}$ after the OER test. Inset :

SAED images of $\mathrm{V}-\mathrm{FeNi}_{2} \mathrm{P} / \mathrm{NF}$ after the OER test. 
Table S4. Water splitting reversibility data based on $\mathrm{V}-\mathrm{FeNi}_{2} \mathrm{P} / \mathrm{NF}$ with current densities from 10 to $200 \mathrm{~mA} \mathrm{~cm}^{-2}$ in $1 \mathrm{M} \mathrm{KOH}$.

\begin{tabular}{|c|c|c|c|}
\hline $\mathrm{j}\left(\mathrm{mA} \mathrm{cm}^{-2}\right)$ & $\eta(\mathrm{mV})$-start & $\eta(\mathrm{mV})$-end & $|\triangle \mathrm{E}|(\mathrm{mV})$ \\
\hline 10 & 1.582 & 1.584 & 0.002 \\
\hline 20 & 1.644 & 1.642 & 0.002 \\
\hline 50 & 1.736 & 1.749 & 0.013 \\
\hline 100 & 1.801 & 1.805 & 0.004 \\
\hline 200 & \multicolumn{2}{|c|}{1.908} & I \\
\hline
\end{tabular}


(a)

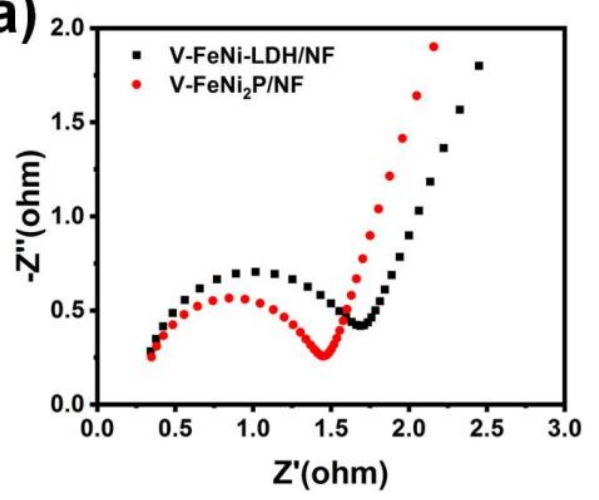

(b)

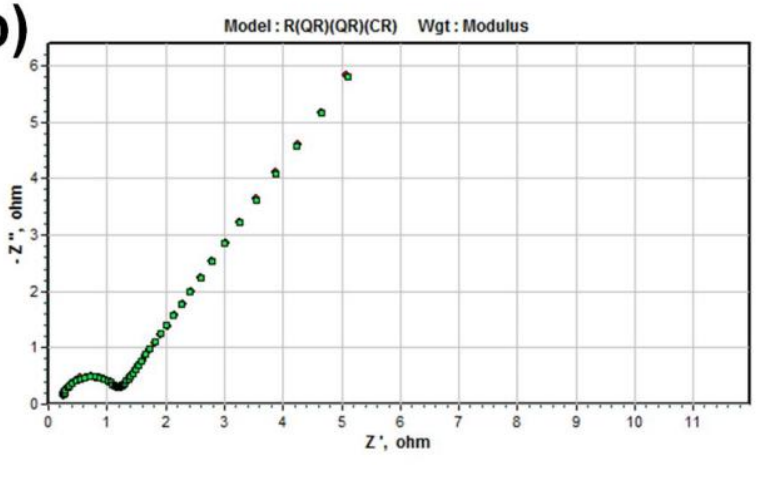

Figure S16. (a) Nyquist electrochemical impedance spectra of $\mathrm{V}-\mathrm{FeNi}_{2} \mathrm{P} / \mathrm{NF}$ and $\mathrm{V}-\mathrm{FeNi}-\mathrm{LDH} / \mathrm{NF}$.

(b) Equivalent circuit model for $\mathrm{V}-\mathrm{FeNi}_{2} \mathrm{P} / \mathrm{NF}$.
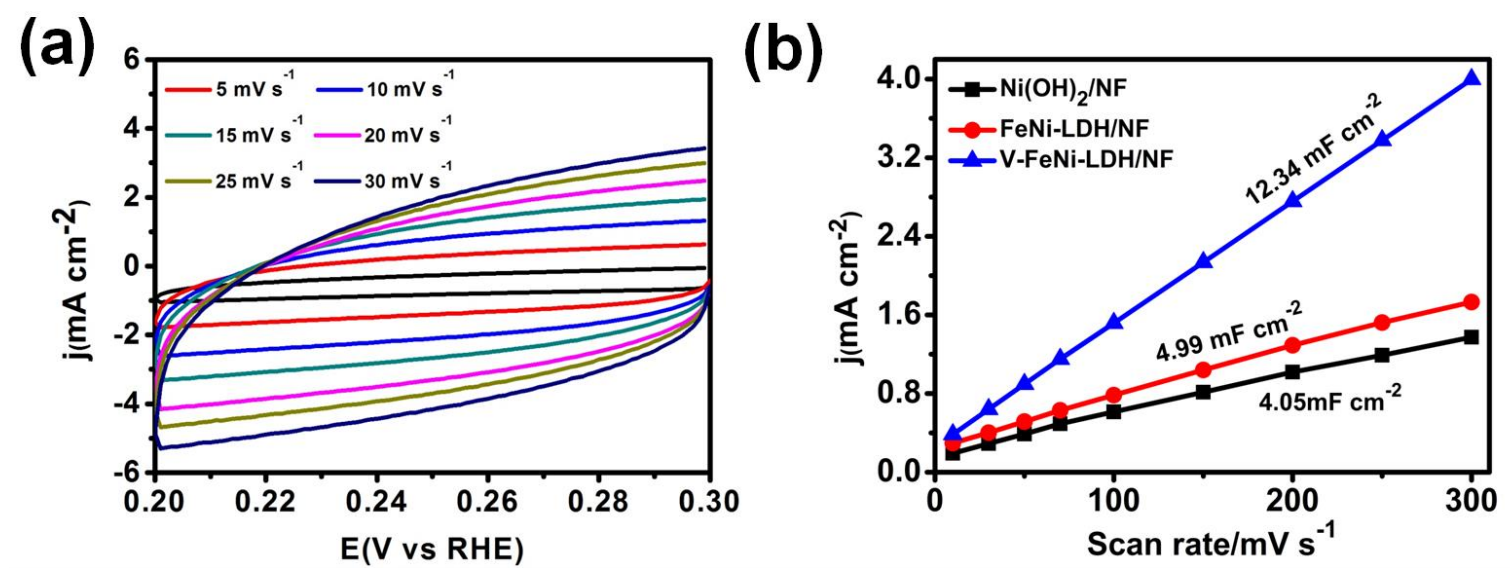

Figure S17. (a) Cyclic voltammograms measured for the $\mathrm{V}-\mathrm{FeNi}_{2} \mathrm{P} / \mathrm{NF}$ catalysts in $1 \mathrm{M} \mathrm{KOH}$ at different scan rates from 10 to $300 \mathrm{mV} \mathrm{s}^{-1}$. (b) $\mathrm{C}_{\mathrm{dl}}$ Plots of $\mathrm{Ni}(\mathrm{OH})_{2} / \mathrm{NF}, \mathrm{FeNi}-\mathrm{LDH} / \mathrm{NF}$ and V-FeNi-LDH/NF.

\section{REFERENCES}

(1) Sun, H.; Min, Y. X.; Yang, W. J.; Lian, Y. B.; Lin, L.; Feng, K.; Deng, Z.; Chen, M. Z.; Zhong, J.; Xu, L.; Peng, Y. Morphological and electronic tuning of $\mathrm{Ni}_{2} \mathrm{P}$ through iron doping towards highly efficient water splitting. ACS Catal. 2019, 9, 8882-8892. 
(2) Yan, H. J.; Xie, Y.; Wu, A. P.; Cai, Z. C.; Wang, L.; Tian, C. G.; Zhang, X. M.; Fu, H. G. Anion-modulated HER and OER activities of 3D Ni-V-based interstitial compound heterojunctions for high-efficiency and stable overall water splitting. Adv. Mater. 2019, 1901174.

(3) Yang, Z.; Lin, Y.; Jiao, F. X; Li, J. H.; Wang, J. L.; Gong, Y. Q. In situ growth of 3D walnut-like nano-architecture $\mathrm{Mo}^{-\mathrm{Ni}}{ }_{2} \mathrm{P} @ \mathrm{NiFe} \mathrm{LDH} / \mathrm{NF}$ arrays for synergistically enhanced overall water splitting. J. Energy Chem. 2020, 49, 189-197.

(4) Wen, L. L.; Yu, J.; Xing, C. C.; Liu, D. L.; Lyu, X. J.; Cai, W. P.; Li, X. Y. Flexible Vanadium-doped $\mathrm{Ni}_{2} \mathrm{P}$ nanosheet arrays grown on carbon cloth for efficient hydrogen evolution reaction. Nanoscale 2019, 11, 4198-4203.

(5) Weng, B. C.; Wang, X. M.; Grice, C. R.; Xu, F. H.; Yan, Y. F. A new metal-organic open framework enabling facile synthesis of carbon encapsulated transition metal phosphide/sulfide nanoparticle electrocatalysts. J. Mater. Chem. A 2019, 7, 7168-7178.

(6) Li, M.; Li, S.; Wang, J. L.; Wang, C. R.; Li, W.; Chu, P. K. NiFeP nanoflakes composite with $\mathrm{CoP}$ on carbon cloth as flexible and durable electrocatalyst for efficient overall water splitting. Nanotechnology 2019, 30, 485402.

(7) Xu, P.; Qiu, L. J.; Wei, L. C.; Liu, Y. L.; Yuan, D. S.; Wang, Y.; Tsiakaras, P. Efficient overall water splitting over $\mathrm{Mn}$ doped $\mathrm{Ni}_{2} \mathrm{P}$ microflowers grown on nickel foam. Catal. Today 2019. 
(8) Lin, J. H.; Wang, H. H.; Yan, Y. T.; Cao, J.; Qu, C. Q.; Zheng, X. H.; Feng, J. C.; Qi, J. L. Sandwich-like structured $\mathrm{NiSe}_{2} / \mathrm{Ni}_{2} \mathrm{P} @ \mathrm{FeP}$ interface nanosheets with rich defects for efficient electrocatalytic water splitting. J. Power Sources 2020, 445, 227294.

(9) Li, R.-Q.; Wang, B.-L.; Gao, T.; Zhang, R.; Xu, C. Y.; Jiang, X. F.; Zenga, J. J.; Bando, Y.; Hu, P. F.; Li, Y.L.; Wang, X.-B. Monolithic electrode integrated of ultrathin NiFeP on 3D strutted graphene for bifunctionally efficient overall water splitting. Nano Energy 2019, 58, 870-876.

(10) Bu, F.-X.; Chen, W. S.; Aly Aboud, M. F.; Shakir, I.; Gu, J. J.; Xu, Y. X. Microwave-assisted ultrafast synthesis of adjustable bimetal phosphide/graphene heterostructures from MOFs for efficient electrochemical water splitting. J. Mater. Chem. A 2019,7, 14526-14535.

(11) Bian, J. L.; Song, Z. Y.; Li, X. L.; Zhang, Y. Z.; Cheng, C. W. Nickel iron phosphide ultrathin nanosheets anchored on nitrogen-doped carbon nanoflake arrays as a bifunctional catalyst for efficient overall water splitting. Nanoscale 2020, 12, 8443-8452.

(12) Xi, W. G.; Yan, G.; Lang, Z. L.; Ma, Y. Y.; Tan, H. Q; Zhu, H. T; Wang, Y. H.; Li, Y. G. Oxygen-Doped nickel iron phosphide nanocube arrays grown on ni foam for oxygen evolution electrocatalysis. Small 2018, 14, 1802204.

(13) Sun, Y. Q.; Zhang, T.; Li, X. Y.; Bai, Y.; Lyu, X. J.; Liu, G. Q.; Cai, W. P.; Li, Y. Bifunctional hybrid $\mathrm{Ni} / \mathrm{Ni}_{2} \mathrm{P}$ nanoparticles encapsulated by graphitic carbon supported with $\mathrm{N}, \mathrm{S}$ 
modified 3D carbon framework for highly efficient overall water splitting. Adv. Mater. Interfaces 2018, 5, 1800473.

(14) Tian, L.; Wo, H. X.; Wang, K.; Wang, X.; Zhuang, W. C.; Li, T. X.; Du, X. H. Ultrathin wrinkled NiFeP nanosheets enable efficient oxygen evolution electrocatalysis. J. Taiwan Inst. Chem. E. 2019, 97, 200-206.

(15) Ji, P. X; Jin, H. H; Xia, H. J.; Luo, X.; Zhu, J. K; Pu, Z. H; Mu, S. C. Double metal diphosphide pair nanocages coupled with P-doped carbon for accelerated oxygen and hydrogen evolution kinetics. ACS Appl. Mater. Interfaces 2020, 12, 727-733. 